\title{
PPP Model Applicability Evaluation of Sewage Treatment Project Based on the Dual Perspective of Government and Private Sector
}

\author{
Ren Xiaoqiang ${ }^{1}$, Chen Ranran ${ }^{2}$ \\ ${ }^{1}$ Power China Zhongnan Engineering Corporation Limited, Changsha 410014 \\ ${ }^{2}$ Institute of Hohai University, Nanjing 211100
}

\begin{abstract}
At present, the applicability evaluation perspective of PPP mode of sewage treatment project in China is single, and the operability of evaluation method is poor. On the basis of the existing evaluation methods, combined with the characteristics of China's sewage treatment projects, this paper establishes the applicability evaluation model of PPP mode of sewage treatment projects from the dual perspectives of government and private sectors, and introduces the psc-ppp value comparison method and the marketability theory to evaluate the value for money. The research results show that the method is feasible and has practical reference value for the applicability evaluation of PPP mode in sewage treatment projects.
\end{abstract}

\section{1 introduction}

With the rapid development of urbanization and industrialization in China, the infrastructure construction in the field of sewage treatment is developing rapidly. In recent years, the state has paid more and more attention to sewage treatment projects, and the PPP model is considered to be the most effective way to introduce social capital into infrastructure construction. Consider introducing the PPP model into sewage treatment construction projects, which will buffer the government's financial pressure on sewage treatment construction. Although sewage treatment projects are infrastructure construction projects, their construction and operation must first consider the interests of the public and society. However, the nature of the sewage treatment industry ensures the sustainable profitability and continuous demand of sewage treatment projects. The characteristics are very attractive to the participation of the private sector, indicating that the PPP model has the potential to be used in the field of sewage treatment. As the sewage treatment project is an infrastructure project that the government strongly supports, the government prefers sewage treatment projects in terms of policy support and financial subsidies.

At present, the application cases of PPP procurement mode in sewage treatment projects in China continue to increase. Scholars in relevant water conservancy fields and PPP fields are also increasingly studying the application of PPP mode in the field of sewage treatment, including studies on the applicability of PPP mode in sewage treatment projects ${ }^{[1,2]}$. However, these studies have certain defects. For lack of guiding significance to practical application, further led to the current application of PPP model in our country's lack of sewage treatment project of science evaluation applicability of PPP model ${ }^{[3-6]}$ PPP model applicability is whether to choose the PPP project procurement mode of important judgment basis, judgment improper or even lack of judgment to government departments and the private sector involved in PPP projects have great risk Based on this, this paper studies the applicability of PPP model for sewage treatment projects, and establishes the applicability evaluation model of PPP model from the dual perspectives of the government and the private sector, so as to bring a more referential evaluation method for the applicability of PPP model for projects.

The applicability evaluation of PPP mode is the premise of whether the project adopts PPP mode. Yuan Jingfeng et al. [7] combined with the specific characteristics of China's projects and the development status of PPP mode in China, applied the value for money theory to the evaluation of China's affordable housing PPP projects; Wang Shouqing ${ }^{[8]}$ made a more specific study on the PSC evaluation system in value for money, adjusted the composition of PSC evaluation index combined with the actual situation of China's projects. ${ }^{[9]}$ analyzed the research and application of several typical foreign countries in the field of value for money, and concluded that the current value for money evaluation methods are mainly cost-benefit analysis method and PSC comparison method; Grimsey et al. ${ }^{[10]}$ analyzed the selection of discount rate in the process of value for money calculation and PS This paper studies the composition and calculation of PSC, and considers that it is necessary and subjective to apply PSC in value for money evaluation. Shaoul ${ }^{[11]}$ proposes that it is unscientific to determine VFM only by the adjustment of government funds. Khadaroo [12] studied some hypothetical conditions in the evaluation process of

\footnotetext{
Corresponding author: xuer2399@163.com
} 
VFM, and found that some assumptions were defined too randomly, which led to unreliable results Coulson studies the PSC model in the PPP bidding documents published by the UK Ministry of Finance and finds that the financial model is complicated. He believes that the current PSC evaluation model needs a lot of historical data to support it, this also has an impact on the accuracy of the evaluation results ${ }^{[13-15]}$.

Through the existing research, it is found that the applicability of PPP mode is more macro- level research, and the research on the applicability of PPP mode for specific industries does not reflect the implementation value. In addition, the applicability evaluation methods of PPP mode in China are mainly from the perspective of the government, while the evaluation methods of PPP mode applicability from the perspective of the private sector are lack. In view of the shortcomings of the existing research, this paper uses the research results of the existing evaluation methods for reference to establish the applicability evaluation model of PPP mode of sewage treatment project from the dual perspectives of government and private sector.

\section{Theory of PPP model applicability in wastewater treatment project}

(1) Value for money theory

The basic idea of the concept of value for money (VFM) is to ensure that the cost of the project in the whole life cycle is minimal and the goal can be achieved. The value difference between the whole life cycle cost (LCC) of traditional purchasing mode and the LCC of PPP mode is called VFM value. The value-for-money method mainly evaluates whether the government department and the private department can obtain the maximum benefit from the operation and management of the whole life cycle of the project, and then determines which procurement method the project adopts according to the evaluation result. Value for money is not only the criterion for evaluating the applicability of PPP projects, but also the most important objective for the government to choose the PPP model.

\section{(2) PSC-PPP comparison method}

PSC (Public Sector Comparator) is the cost of the project under the traditional procurement mode of government. PSC is used as the benchmark price in the evaluation of the applicability of the PPP mode to compare with the PPP cost of the whole life cycle of the PPP project, therefore, the determination of PSC composition and the calculation of PSC value are very important for PPP projects. The components of PSC are defined differently in different countries, but the main components are divided into initial PSC, transfer risk cost, retention risk cost and competitive neutral price.

(3) Marketability theory

The so-called marketability refers to the potential of a product or service, which can be obtained by judging the possibility of the product or service entering the market. Accordingly, the marketability of infrastructure can be obtained by judging the potential of the private sector to provide infrastructure through market mechanism. In view of the non-competitive index, the non-exclusive index, the scale economy index, the SUNK Cost Index, the Coordination Index, as well as the index which sets according to the external effect and the social goal, this paper carries on the qualitative judgment, the evaluation results are divided into high, medium and low standards, and then the six results are analyzed comprehensively to get the salability of the project to be evaluated.

\section{Construction of the applicability evaluation model of PPP project from the perspective of government}

\subsection{Formatting the title, authors and affiliations}

Based on the comparative analysis of PSC composition in different countries and the current research situation in China ${ }^{[17]}$, this paper determines that the main key factors of PSC composition in sewage treatment PPP project mainly include the net cost of construction, operation and maintenance, competitive neutral adjustment value and total risk cost of the project, etc..

(1) The net cost of construction, operation and maintenance V1, also known as the initial PSC ${ }^{[18]}$. The initial PSC of the sewage treatment project refers to the basic cost of the whole life cycle of the sewage treatment project provided by the government, mainly including the cost of investment and financing, project construction, project operation and later maintenance of the sewage treatment project.

(2) Transfer risk cost V2, which is an important part of PSC, is the part of risk transferred from government to private sector after government allows private sector to participate in PPP project. ${ }^{[19]}$.

(3) Retaining risk V 3 is a risk borne by the government itself, which is not transferred to the private sector when the cost of transferring risk is higher than the cost of government's own assumption of risk, this part of the risk is called the retention risk.

(4) The main reason for the existence of V4 is the nature of government departments, which bring competitive advantage to the project. The private sector makes profits from PPP projects, while the public sector raises government taxes through PPP projects. The competitive neutral price of sewage treatment PPP project mainly consists of land cost, income tax, valueadded tax and additional tax.

Through the integration of the above four components, all factors are converted into currency. The PSC of the sewage treatment project can be expressed as:

$$
V_{P S C}=V 1+V 2+V 3+V 4
$$

\subsection{Calculation of PPP for sewage treatment}

The whole life cycle cost of PPP project is the total cost of PPP project in the construction, operation, maintenance and other stages, which is mainly divided into two parts: initial cost and future cost, which are 
specifically manifested as basic cost, risk cost and special cost of PPP ${ }^{[20]}$.

(1) The basic cost C1 of PPP is the basic cost incurred in the whole life cycle of a PPP project. The whole life cycle cost of sewage treatment PPP project includes investment and financing cost,construction cost, operation and maintenance cost, dismantling cost and so on.

(2) The risk cost $\mathrm{C} 2+\mathrm{C} 3$ mainly includes two parts: the private sector's risk cost $(\mathrm{C} 2)$ and the government's risk cost that can't be transferred to the private sector, namely the self-retaining cost (C3).

(3) Special cost $\mathrm{C} 4$, mainly including private financing cost, risk transfer cost, supervision cost and transaction cost, etc..

Based on the above analysis of the composition of LCC, the formula for calculating the PPP value is as follows:

$$
V_{P P P}=C 1+C 2+C 3+C 4
$$

\subsection{Applicability judgment of sewage treatment PPP project}

Combined with the composition of PSC value and PPP value, the calculation formula of VFM value of PPP project for sewage treatment is obtained as follows: $V_{V F M}=V_{P S C}-V_{P P P}=(V 1+V 2+V 3+V 4)-(C 1+C 2+C 3+C 4)$

In order to ensure the comparability of the PSC value and the PPP value in the government procurement mode, the discount rate and the reference date should be the same standard. The exact composition of the VFM value is shown in Fig. 1.

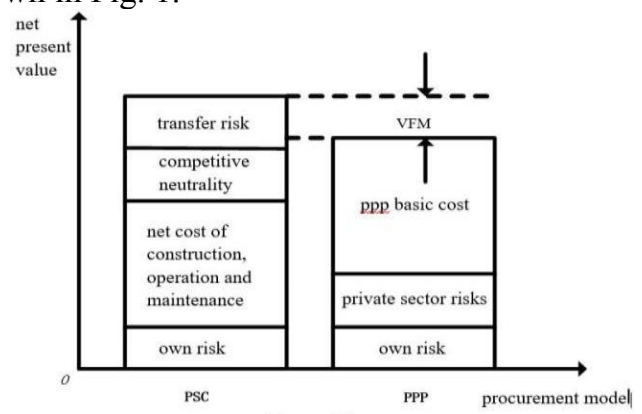

Fig. 1. VFM composition.

The relationship between VFM value and PPP mode of sewage treatment project is as follows:

(1) By comparing the PSC value and PPP value calculated respectively, when there is a difference between the PSC value and PPP value in Figure 3.1, namely VFM value, it means that the PSC value under the government procurement mode is more than the PPP value under the PPP procurement mode. At this time, it can be judged that the project is suitable for the PPP mode, and the applicability of the PPP mode can be further obtained according to the VFM value, It should be judged according to the characteristics of the project and the negotiation between the government and the private sector.

(2) When the difference between PSC value and PPP value is small, the method chosen in this paper is to judge PPP applicability from the existing government perspective. When the result of qualitative index evaluation is that the applicability of PPP mode is higher, and the VFM value can be accepted by both parties through negotiation between the government and the private sector, the PPP procurement mode can be adopted; If the result of qualitative index analysis is that PPP mode is not suitable or the advantages of both purchasing modes are not obvious, and the acceptance degree of VFM value is not agreed by both sides through negotiation, then the project is judged not to be suitable for PPP procurement mode.

(3) When the PSC value and PPP value are calculated, it is found that the former is significantly higher than the latter, which means that the project is not suitable for PPP mode, and the traditional procurement mode is directly used.

\section{Construction of the applicability evaluation model of PPP project from the perspective of private sector}

\subsection{Evaluation index selection and weight determination}

This article takes into account the operability of the evaluation model and the accuracy of the evaluation results, and selects the first five indicators to construct the model, including competitiveness, exclusivity, sunk costs, economies of scale, and coordination. Externalities and social benefits, which can be used as supplementary indicators, are not within the scope of this model.

The weight of each index is determined by twodimensional quantitative method. In this paper, 10 experts in the field of water conservancy and PPP are selected to score the importance of the applicability evaluation index of PPP mode of sewage treatment project by questionnaire survey method, and then the importance level variable is obtained by ranking according to the score results, and the importance of the index is expressed by "very important, important, generally important and unnecessary" 3, 2, 1 and 0 respectively. Finally, the weights of qualitative indicators for PPP mode applicability of sewage treatment projects are: $\eta 1$ (competitiveness) $=0.299 ; \eta$ $2($ exclusiveness $)=0.358 ; \eta 3($ sunk cost $)=0.201 ; \eta 4$ $($ economies of scale $)=0.063 ; \eta 5($ coordination $)=0.079$.

\subsection{Evaluation model construction}

This paper selects the gray cluster evaluation method to construct the evaluation model of the qualitative index of value for money.

\section{(1) Evaluation system establishment}

The evaluation object is represented by $i$, the evaluation index is represented by $\mathrm{j}$, and the evaluation gray class is represented by $\mathrm{k}$. The gray class evaluation system can be expressed as $\{\mathrm{I}, \mathrm{J}, \mathrm{K}\}$. I represents the evaluation object set composed of the object to be evaluated $\mathrm{i}, \mathrm{J}$ represents the evaluation index set composed of evaluation index $\mathrm{j}$, and $\mathrm{K}$ represents the 
evaluation gray class set composed of evaluation gray class $\mathrm{k}$; among them, the evaluation gray class set $\mathrm{K}$ is divided into high degree of applicability and applicable There are four evaluation gray categories: high degree, medium degree of application, and low degree of application.

\section{(2)Evaluation matrix establishment}

The value of the evaluation matrix is composed of the results obtained by evaluating each index value of the item to be evaluated. In this paper, the expert scoring method is used to score the item to be evaluated, and the average value of the score forms each value of the evaluation moment.

Let the sample to be evaluated be I $(I=1,2 \mathrm{n})$, and the evaluation index $\mathrm{J}(\mathrm{J}=1,2 \mathrm{~m}$. If is the observation value of object I about index $\mathrm{J}$, forms the evaluation sample matrix X.

$$
\mathrm{X}=\left(\begin{array}{cccc}
x_{11} & x_{12} & \ldots & x_{1 \mathrm{~m}} \\
x_{21} & x_{22} & \ldots & x_{2 m} \\
\ldots & \ldots & & \ldots \\
x_{n 1} & x_{n 2} & \ldots & x_{n m}
\end{array}\right)=\left(x_{i j}\right)_{n m}
$$

\section{(3) Evaluation grey class setting}

1) Let the evaluation grey class be $K(k=1,2 \mathrm{~s})$ This paper is to evaluate the applicability of PPP mode of sewage treatment project. Because the applicability degree is divided into four degrees in this paper, the grey value is 4 . It indicates high, high, medium and low applicability respectively.

2) Determine the whitening weight function. The whiteness weight function of this paper is determined according to the standard of dividing the applicability of PPP mode qualitative evaluation index level of sewage treatment project.

In this paper, the index whitenization weight function is expressed in the form of piecewise function. The concrete expression is: $(\mathrm{j}=1,2, \ldots \mathrm{m}, \mathrm{M}=5, \mathrm{k}=1,2, \ldots \mathrm{s}$, $\mathrm{S}=4)$. Here, the grey class is quantified as $0-10$, the whitening weight functions for each sub-category of each indicator are ultimately determined as follows:

First, competitiveness and exclusivity $(j=1,2)$

$$
\begin{aligned}
& f_{j}^{1}=(7,9.5, \quad-, \quad-) ; \quad f_{j}^{2}=(5,6, \quad-, 7) ; \\
& f_{j}^{3}=(3,4.5, \quad-, \quad 5) ; \quad f_{j}^{4}=(-, \quad-, \quad 1,3) ;
\end{aligned}
$$

Second, sunk costs and economies of scale $(j=3,4)$

$$
\begin{aligned}
& f_{j}^{1}=(-, \quad-, \quad 4,6.5) ; \quad f_{j}^{2}=(6.5, \quad 7.5, \quad-, 8.5) ; \\
& f_{j}^{3}=(8.5, \quad 9,-, \quad 9.5) ; \quad f_{j}^{4}=(9,10, \quad-, \quad-) ;
\end{aligned}
$$

Third, compatibility $(\mathrm{J}=5)$

$$
\begin{aligned}
& f_{j}^{1}=(6,9.5, \quad-, \quad-) ; f_{j}^{2}=(4,5, \quad-, 6) ; \\
& f_{j}^{3}=(3,4.5, \quad-, \quad 5) ; \quad f_{j}^{4}=(-, \quad-, \quad 1,3) ;
\end{aligned}
$$

(4) Calculating the clustering coefficient

The clustering coefficient represents the degree of membership of the clustering samples. The data involved in the calculation of clustering coefficient are whitening weight function $f_{j}^{k}().(\mathrm{j}=1,2, \ldots \mathrm{m}, \mathrm{m}=5, \mathrm{k}=1,2, \ldots \mathrm{s}$, $\mathrm{s}=4)$, clustering weight ${ }_{\eta_{j}}$, observation value $\left(\mathrm{i}_{x_{i j}}=1,2, \mathrm{n}\right.$; $\mathrm{j}=1,2, \cdots, \mathrm{m}, \mathrm{m}=5)$. The calculation formula of clustering coefficient is:

$$
\sigma_{\mathrm{ik}}=\sum_{j=1}^{m} f_{j}^{k}\left(x_{i j}\right) \eta_{\mathrm{j}}(\mathrm{i}=1,2, \mathrm{n} ; \mathrm{k}=1,2, \cdots \mathrm{s}, \mathrm{s}=4) .
$$

\subsection{PPP project suitability judgment from the perspective of private sector}

The judgment of grey class of clustering samples is based on the maximum coefficient of clustering vector. The clustering vector composition of sample I to be evaluated is $\sigma_{i}=\left[\sigma_{i 1}, \sigma_{i 2}, \ldots, \sigma_{i k}\right]$, if $\sigma_{i}^{k^{*}}=\max \left\{\sigma_{i 1}, \sigma_{i 2}, \ldots, \sigma_{i k}\right\}$, then the cluster sample I belongs to the Grey Class $\mathrm{K} *$.

According to the result of model, the applicability of sewage treatment PPP can be classified, and the result of classification is the applicability of PPP model. The private sector can use this as a basis for deciding whether or not to participate in infrastructure projects.

\section{Case studies}

\subsection{Project background}

A sewage treatment plant $\mathrm{M}$ in City A was invested and constructed in 2000 and put into operation in 2004. The main projects of the sewage treatment plant are environmental protection projects. Limited to factors such as insufficient funds for municipal construction by the municipal government of City A, the government adopt the PPP model to construct the sewage treatment plant upgrade project. The planned construction period is two years. The total construction scale of the project is 100,000 , and the wetland area is 43 hectares. The cooperation period between the government and the private sector is 25 years. The total project investment is 130 million yuan, and the unfinished part of the project is 80 million yuan. The investment cost recovery method is "government payment", and the corresponding profit is obtained by charging government fees. The life cycle cost of the project includes the remaining construction cost of 80 million yuan, the operation and maintenance cost of 2,856,500 yuan and related tax expenses. At the same time, if the consumer price index increases by more than $5 \%$ due to the labor market index, the private sector can apply for adjustment of the service fee price in accordance with the procedures. The main technical and economic indicators of the project are shown in Table 1 .

Table 1. Project investment costs and indicators.

\begin{tabular}{|c|c|c|c|c|}
\hline & \multicolumn{2}{|c|}{ Technical specification } & \multicolumn{2}{c|}{ Economic Indicators (RMB) } \\
\hline 1 & Amount of treated water & $\begin{array}{c}100,000 \mathrm{~m}^{3} \\
/ \mathrm{d}\end{array}$ & $\begin{array}{c}\text { Investment } \\
\text { estimation }\end{array}$ & 12818 \\
\hline 2 & Annual treated water $\mathrm{m}^{3} / \mathrm{d}$ & 3650 & $\begin{array}{c}\text { Construction } \\
\text { cost }\end{array}$ & 8000 \\
\hline
\end{tabular}




\begin{tabular}{|c|c|c|c|c|}
\hline 3 & $\begin{array}{c}\text { Area of subsurface flowwet-land } \\
\text { (hectare) }\end{array}$ & 22 & Capital gains & 400 \\
\hline 4 & $\begin{array}{c}\text { Surface flow wetland area } \\
\text { (hectare) }\end{array}$ & 6.45 & $\begin{array}{c}\text { Annual } \\
\text { operating cost }\end{array}$ & 286.65 \\
\hline 5 & Fixed member (person) & 14 & & \\
\hline 6 & Installed capacity (kw) & 230 & & \\
\hline 7 & $\begin{array}{c}\text { Electricity-based load calculation } \\
(\mathrm{kw})\end{array}$ & 165 & & \\
\hline 8 & $\begin{array}{c}\text { Annual electricity consumption } \\
(\mathrm{kwh})\end{array}$ & 143.67 & & \\
\hline 9 & Power consumption $\left(\mathrm{kwh} / \mathrm{m}^{3}\right)$ & 0.04 & & \\
\hline 10 & Bod reduction (t/a) & 7.30 & & \\
\hline 11 & Cod reduction $(\mathrm{t} / \mathrm{a})$ & 385 & & \\
\hline 12 & NH5-N reduction $(\mathrm{t} / \mathrm{a})$ & 219 & & \\
\hline 13 & TP reduction (t/a) & 21.9 & & \\
\hline
\end{tabular}

\subsection{PPP project suitability evaluation from the} perspective of government

\subsubsection{PSC calculation}

The net cost of construction, operation and maintenance of the sewage treatment project is 2.865 million yuan, the competitive neutral price is 10,000 yuan, the transfer risk cost is 400,000 yuan, and the retention risk cost is 142,800 yuan. From the PSC formula of the project, the PSC value of the project is calculated as:

$\mathrm{V}_{\mathrm{PSC}}=\mathrm{C}_{\mathrm{iPSC}}+\mathrm{C} 3+\mathrm{C} 4+\mathrm{A}=20860.44$ (million yuan)

The discount rate is calculated at $4 \%$, and the present value of PSC (NPVPSC) is 15393.64 million yuan.

\subsubsection{PPP value calculation}

The whole life cycle cost (LCC) of the project in PPP mode should be calculated by taking into account the cash inflow and cash outflow of government departments. Based on the above assumptions, the profit rate of the sewage treatment project is calculated and the discount rate is calculated. By calculating the financial cash flow of the project, the static payback period of the project is years, and the dynamic payback period of the investment is years, the internal rate of return is calculated. The net present value (NPVPPP) of the project during the concession period is 12.331 million yuan.

\subsubsection{Project VFM analysis}

Through the calculation and analysis of the full life cycle (LCC) under the PSC and PPP modes of government departments, Table 2 is obtained by comparing the calculation results under the two modes.

Table 2. Price comparison table.

\begin{tabular}{|c|c|c|}
\hline Indicators & Unit & Numerical value \\
\hline NPVPSC & million yuan & 15393.64 \\
\hline NPVPPP & million yuan & 11031.50 \\
\hline VFM value & million yuan & 4362.14 \\
\hline VFM index & $\%$ & $28.34 \%$ \\
\hline
\end{tabular}

It can be seen from the above table that the VFM value of the project is positive.According to the results of VFM quantitative evaluation, it is worth while for the government to adopt PPP mode.

\subsection{Applicability evaluation of PPP projects from the perspective of private sector}

In this paper, four reference projects are selected: a sewage treatment PPP project in Weining, Xuzhou, a sewage treatment PPP project in Nanjing Dachang District, a sewage treatment PPP project in Zhenjiang
Yangzhong, and a sewage treatment PPP project in Yancheng. The process of grey clustering evaluation for these five projects is as follows:

\subsubsection{Evaluation sample collection}

As the five projects are sewage treatment projects, which belong to the category of water conservancy projects, the characteristics of the project should be considered when collecting the sample values. A total of 50 experts in the field of hydraulic engineering from universities and research institutes in Jiangsu province were selected as objects for the questionnaire, which was independently graded with a total score of 10 , the five items in the case were rated on the five indicators by 50 experts. According to the score of each expert, the average value of each index is calculated, and the result is regarded as the sample value of grey clustering evaluation, and the sample matrix is composed. Table 3 is based on the expert scoring results of the final determination of the indicator sample values.

Table 3. Evaluation sample values.

\begin{tabular}{|c|c|c|c|c|c|}
\hline \multirow{2}{*}{ Projects } & \multicolumn{5}{|c|}{ Evaluation Index } \\
\cline { 2 - 6 } & $\begin{array}{c}\text { Comp } \\
\text { etitive } \\
\text { ness }\end{array}$ & $\begin{array}{c}\text { exclusi } \\
\text { veness }\end{array}$ & $\begin{array}{c}\text { Sunk } \\
\text { costs }\end{array}$ & $\begin{array}{c}\text { scale } \\
\text { econo } \\
\text { my }\end{array}$ & $\begin{array}{c}\text { compa } \\
\text { tibility }\end{array}$ \\
\hline 1.M project & 8.14 & 8.5 & 7.9 & 5.90 & 7.25 \\
\hline 2.Nanjin & 6.15 & 5.5 & 7.85 & 5.25 & 8.13 \\
\hline 3. Zhenjiang & 3.5 & 4.35 & 5.76 & 6.20 & 8.24 \\
\hline 4.Yancheng & 7.4 & 9.12 & 7.40 & 8.67 & 6.50 \\
\hline 5.Xuzhou & 4.3 & 7.32 & 8.14 & 6.2 & 4.5 \\
\hline
\end{tabular}

The results are as follows:

$$
X=\left(\begin{array}{ccccc}
8.14 & 8.5 & 7.9 & 5.9 & 7.25 \\
6.15 & 5.5 & 7.85 & 5.25 & 8.13 \\
3.5 & 4.35 & 5.76 & 6.2 & 8.24 \\
7.4 & 9.12 & 7.4 & 8.67 & 6.5 \\
4.3 & 7.32 & 8.14 & 6.2 & 4.5
\end{array}\right)
$$

\subsubsection{Calculation of clustering coefficient}

According to the formula $\sigma_{\mathrm{ik}}=\sum_{j=1}^{m} f_{j}^{k}\left(x_{i j}\right) \eta_{\mathrm{j}}(\mathrm{i}=1,2,3,4,5$;

$\mathrm{K}=1,2,3,4)$ to calculate the cluster coefficient, and then calculate the cluster vector of PPP evaluation in the field of sewage treatment.

$$
\begin{aligned}
& \sigma_{1}=(0.394,0.121,0,0) ; \\
& \sigma_{2}=(0.0796,0.564,0,0) ; \\
& \sigma_{3}=(0.118,0,0.422,0) ; \\
& \sigma_{4}=(0.363,0.181,0.021,0) ; \\
& \sigma_{5}=(0.053,0.112,0.338,0) .
\end{aligned}
$$

According to the clustering coefficient and the clustering vector, the gray fixed-weight clustering coefficient matrix is obtained as follows:

$$
\sigma_{\mathrm{i}}^{\mathrm{k}}=\left[\begin{array}{cccc}
0.394 & 0.121 & 0 & 0 \\
0.0796 & 0.564 & 0 & 0 \\
0.118 & 0 & 0.422 & 0 \\
0.363 & 0.181 & 0.121 & 0 \\
0.053 & 0.112 & 0.338 & 0
\end{array}\right]
$$

\subsubsection{Evaluation sample collection}


From the grey fixed weight clustering coefficient matrix, the following results can be obtained:

$$
\begin{aligned}
& \max _{1 \leq k \leq 4}=\left\{\sigma_{1}^{k}\right\}=\sigma_{1}^{1}=0.394 ; \\
& \max _{1 \leq k \leq 4}=\left\{\sigma_{2}^{k}\right\}=\sigma_{2}^{2}=0.564 ; \\
& \max _{1 \leq k \leq 4}=\left\{\sigma_{3}^{k}\right\}=\sigma_{3}^{3}=0.422 \\
& \max _{1 \leq k \leq 4}=\left\{\sigma_{4}^{k}\right\}=\sigma_{4}^{1}=0.363 ; \\
& \max _{1 \leq k \leq 4}=\left\{\sigma_{5}^{k}\right\}=\sigma_{5}^{3}=0.338
\end{aligned} ;
$$

Based on the above results, the applicability of the PPP model for five sewage treatment projects is classified. Sewage treatment plant $M$ upgrade project and a sewage treatment PPP project in Yancheng belong to the category of higher applicability; the project with higher applicability of PPP model is a sewage treatment PPP project of Nanjing Dachang; the applicability of PPP model is medium The projects are a sewage treatment PPP project in Yangzhong, Zhenjiang and a sewage treatment PPP project in Weining, Xuzhou. Since the reference projects selected in this case were constructed using the PPP model and achieved a certain degree of value for money, it can be judged that the upgrade project of the sewage treatment plant $\mathrm{M}$ is suitable for adopting the PPP model based on the evaluation results.

\subsection{Applicability evaluation of PPP projects}

From the government's point of view, the evaluation using the PSC-PPP method shows that the PPP model is more cost-effective than the traditional procurement model for the Sewage Treatment upgrade project. From the perspective of the private sector, compared with other similar projects, the PPP model of this project is more suitable than other projects. The results of the two evaluation methods both indicate that the project can adopt PPP mode. Therefore, the upgrade project of the sewage treatment plant $\mathrm{M}$ is suitable for the PPP procurement model.

\section{Conclusion}

The applicability evaluation model of PPP project from the perspective of government departments proposed in this paper only considers quantitative evaluation, but only mentions qualitative evaluation and is not included in the evaluation model of this paper. This will affect the accuracy of the applicability evaluation of PPP project, which will become the direction of the further research.

\section{References}

1. W L T Q S. A review of studies on Public-Private Partnership projects in the construction industry, International Journal of Project Management,(2010)

2. Demirag I. Accountability and value for money:a theoretical framework for the relationship in publicprivate partnership,Journal of Management and Governance , 2,(2011)
3. Di wu, Wang Shouqing. The research development and trend of PPP model in China. Journal of Engineering Management,20-28,(2014)

4. Rossi M, Civitillo R. Public Private Partnerships: A General Overview in Italy, Procedia - Social and Behavioral Sciences, 140-149,(2014)

5. Gurgun A P, Alitouran. Public-Private Partnership Experience in the International Arena: Case of Turkey, J. Manage, 2-7,(2014)

6. Heald D. Value for money tests and accounting treatment in PFI schemes,Accounting Auditing and Accountability Journal, 3,(2013)

7. Chang Z. Public-private partnerships in China: A case of the Beijing No.4 Metro line, Transport Policy,153-160,(2013)

8. Mao Yanling, $\mathrm{Fu}$ Chun. Application of PPP financing model in rural hydropower development, Water Conservancy and hydropower in rural areas of China, 12,(2009)

9. Bao Chunsheng. Research on innovative mechanism of rural water conservancy facilities construction based on PPP financing model, Water Conservancy in China, 15,(2012)

10. Sobhiyah M H, Bemanian M R. Increasing VFM in PPP power station projects - Case study: Rudeshur gas turbine power station, International Journal of Project Management,27,(2009)

11. Roehrich J K, Lewis M A. Are public-private partnerships a healthy option? A systematic literature review, Social Science \& Medicine,113,(2014)

12. Satterthwaite D. The European Commission. Guidance For Successful PPP, Earthscan Publications Ltd, London,(2011)

13. Cheung E, Chan A P C. Evaluation Model for Assessing the Suitability of Public-Private Partnership Projects, Journal of Management in Engineering,2,,(2011)

14. Love P E D, Liu J, Matthews J. Future proofing PPPs: Life-cycle performance measurement and Building Information Modelling, Automation in Construction,56,26-35,(2015)

15. Clifton C, Duffield C F. Improved PFI/PPP Service Outcomes Through the Integration of Alliance Principles, International Journal of Project Management, 24,(2011)

16. Geddes R R, Wagner B L. Why do U.S. states adopt public - private partnership enabling legislation? Journal of Urban Economics, 78,30-41,(2013)

17. Li Jiarong. Research on PSC evaluation system based on China's national conditions. Tsinghua University,(2011)

18. Diao Fi, Li Feng. Research on application of PSC evaluation method in China's public housing PPP project , Construction economy,36 87-90,(2015) 
19. Guo Shang,Research on the value-for-money evaluation of PPP model in China, Institute of Fiscal Science, Ministry of Finance,(2015)

20. Min Hongdong. Application of value for money quantitative evaluation in PPP project, Financial economy,6,82-84,(2016) 